\title{
Adaptive and Unconventional Strategies for Engine Knock Control
}

\author{
Donald Selmanaj, Giulio Panzani, Stijn van Dooren, Jonatan Rosgren and Christopher Onder
}

\begin{abstract}
Knock is an undesirable phenomenon affecting gasoline spark-ignition (SI) engines. In order to maximize engine efficiency and output torque while limiting the knock rate, the spark timing should be adequately controlled. This paper focuses on closed-loop knock control strategies. The proposed control strategies, compared to conventional approaches, show improved performances while remaining simple to use, implement, and tune. Firstly, a deterministic controller which employs a logarithmic increase of the spark timing proves to outperform the conventional strategy in terms of spark timing average and variance. In addition, an adaptive parameter strategy which exploits stochastic information of the process is introduced. Thanks to this extension the average and the variance of the spark timing are additionally improved while preserving ease of tuning and the fast reaction times of the deterministic strategy. Throughout the paper all the knock controllers are compared with a conventional deterministic strategy and with a recently proposed stochastic one. The advantages of the proposed approaches are confirmed both by simulation and by experimental data collected at a test bench.
\end{abstract}

Index Terms-knock, control, adaptive, stochastic, SI engine

\section{NOMENCLATURE}

\section{A. Acronyms}

bTDC Before Top Dead Centre.

MAPO Maximum Amplitude of Pressure Oscillations, used to detect an engine knocking cycle.

[bar]

\section{B. Symbols}

$S \quad$ Spark timing.

$\left[{ }^{\circ} \mathrm{bTDC}\right]$

$K_{\text {ret }} \quad$ Spark timing reduction for a knocking cycle. $\left[^{\circ}\right]$

$K_{a d v} \quad$ Spark timing increase for a non-knocking cycle. $\left[{ }^{\circ}\right]$

$P_{\text {ref }} \quad$ Target knock probability.

$[0 . .1]$

$j \quad$ Engine cycle counter.

$\mathcal{L}_{r} \quad$ Likelihood ratio.

\section{Subscripts}

c referred to the conventional control strategy.

This project has received funding from the European Union's Horizon 2020 research and innovation programme. Agreement No: 634135-HERCULES-2.

D. Selmanaj is with the Department of Automation, Polytechnic University of Tirana, Sheshi "Nënë Tereza", Nr.4, Tirana, Albania (e-mail: donald.selmanaj@fie.upt.al).

G. Panzani is with the Dipartimento di Elettronica, Informazione e Bioingegneria, Politecnico di Milano, Piazza L. da Vinci 32, Milano, Italy (e-mail: giulio.panzani@polimi.it).

S. van Dooren and C. Onder are with the Institute for Dynamic Systems and Control, ETH Zurich, Sonneggstrasse 3, CH-8092 Zurich, Switzerland (e-mail: stijnva@ethz.ch, onder@idsc.mavt.ethz.ch).

J. Rosgren is with Wärtsilä Finland Oy, FIN-65101, Vaasa, Finland (e-mail: jonatan.rosgren@wartsila.com). $u \quad$ referred to the unconventional control strategy.

ac referred to the adaptive conventional control strategy.

au referred to the adaptive unconventional control strat-

egy.

$k \quad$ referred to the knock event.

th referred to a threshold value.

\section{INTRODUCTION}

Engine knock has its name from the audible noise that results from autoignition in the unburned part of the gas. Causing undesired and abnormal pressure oscillations during the fuel combustion, this phenomenon is a major limitation for SI engines. To avoid engine knocking, in addition to limiting the compression ratio and lowering pressure and temperature levels, the engine has to be run in a suboptimal way - w.r.t. efficiency or delivered engine torque - for example delaying the spark timing from its optimal value [1], [2]. Closed-loop knock control systems acting on spark timing confront the trade-off between knock rate limitation and engine performance maximization.

While considerable research efforts have been dedicated to the problem of knock detection and description [3]-[14], knock control strategies have received less attention. A conventional and widely used in industrial applications strategy consists in rapidly retarding the spark timing if a knock event is observed and slowly advancing it during non-knocking cycles [15]. This strategy is also referred to as deterministic since it acts at each knock event, disregarding the highly random behaviour of the knock phenomenon.

Alternative solutions consist of modelling and controlling statistical properties of knock and for this reason they are usually referred to as stochastic. The majority of the scientific literature approaches the problem using some indirect metrics to describe the knock event. One possibility is to quantify its intensity through engine case accelerations and to build a knock energy indicator which is regulated via a proportional integral (PI) controller [16]. The method requires the estimation of the mean and the variance of the acceleration signal energy, which slows the controller action. Similar approaches based on Ion current measures [17] and combustion indicators [2], [18] aim at improving controller responses by adding fast control actions.

A different stochastic philosophy neglects the knock intensity information and focuses only on statistic properties of knock occurrence [15]. In this way, the description of the knock phenomenon is simplified, as knock events can be easily modelled by standard statistical (e.g., binomial) distributions. 
Following this philosophy a controller that monitors the cumulative summation of knock events and compares it with a desired rate is proposed in [19]: the controller does not act at each knock event but it retards/advances the spark timing when the difference between the observed and the desired knock rate exceeds a threshold. Additional improvements of this method are presented in [20], where the likelihood ratio serves as indicator of the discrepancy between the observed and the desired knock occurrence distribution and it is used to modulate the action of the previously mentioned controller. When compared to deterministic strategies, this approach shows good results on both simulation and experimental data [21]. Although effective, stochastic knock controllers require non-trivial tuning procedures and have an overall delayed transitory response, given that statistic knock properties have to be estimated in real time.

Other recent methods are based on the concept of margin (or distance) from the knocking condition. They relate knock occurrence to measurement data also during non-knocking cycles. One simple example is the peak pressure [22]: cycles with higher peak pressures are more likely to result in knocking ones. Exploiting the same philosophy, in [23], the authors build a gray-box model of the knock margin that proves effective in estimating the actual knock rate in various engine operating conditions, outperforming more traditional physics-based approaches. Despite the lack of an intensive experimental validation of closed-loop control strategies, such approaches require a considerable modelling effort and do not consider engine ageing, which also can change the relation between measurement data and knock occurrence.

In this work three strategies are proposed, aiming at improving both deterministic and stochastic controllers. The first method introduces a logarithmic advance of the spark timing during non-knocking cycles. The second and third method are adaptive strategies that combine the advantages of deterministic controllers (i.e., fast action, implementation and ease of tuning) with the enhanced performance of stochastic approaches. The methods are tested and compared on an engine-validated knock stochastic simulator and at an engine test bench, outperforming both the conventional and the benchmark stochastic strategy [20].

The paper is organized as follows: Section II gives an overview on the knock phenomenon and its stochastic modelling. Section III proposes a new deterministic controller. Section IV introduces the full adaptive scheme combining deterministic controllers with a stochastic adaptation of their parameters. In Section V and Section VI, the simulation and experimental results are shown and discussed.

\section{Stochastic Knock Modelling}

The most evident effect of knock occurrence are the pressure oscillations in the combustion chamber, whose amplitude is strongly related to the knock intensity. Such oscillations can be isolated by properly band-pass-filtering the pressure signal and used to establish various knock metrics: here the MAPO is considered [24]. This metric is the one most commonly used due to its easy implementation and its tight correlation with knock intensity. Two examples of in-cylinder pressures recorded during a knocking and a non-knocking combustion cycle are shown in Figure 1: the pressure oscillations due to knock are clearly detectable with a band-pass filter.

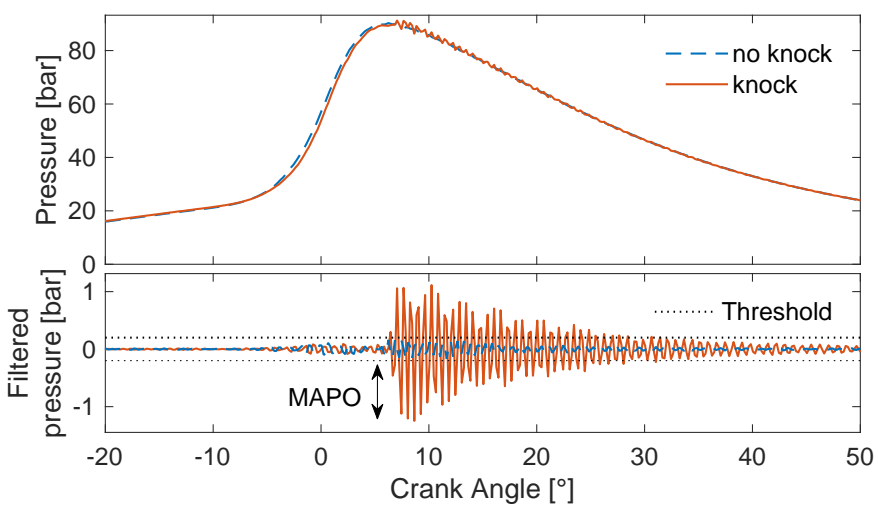

Figure 1. Raw and band-pass-filtered pressure traces and MAPO index for a knocking and a non-knocking cycle.

A knock event is detected when the MAPO exceeds a given threshold. A large threshold value reduces the knock detection sensitivity and recognizes as knocking ones only the cycles with large pressure oscillations, while a small value increases the detection sensitivity, but could lead to an excessive responsiveness since more cycles are treated as knocking ones. For the experimental test bench used in this work, a threshold value of 0.2 bar is found to be a good compromise.

The adopted in-cylinder pressure detection algorithm is not a mandatory requirement for the effective implementation of the knock controllers proposed in the following. Any other method capable of detecting knock occurrence (not its intensity) can be alternatively used, e.g. [4], [25].

The static model which provides the average knock rate as a function of constant spark timing is shown in Figure 2. Given a desired knock rate, the static model provides an indication of the average achievable spark timing. Although a proper controller design and tuning can advance the average spark timing and increase the engine efficiency for a given knock rate, it is physically limited by the characteristics of the engine.

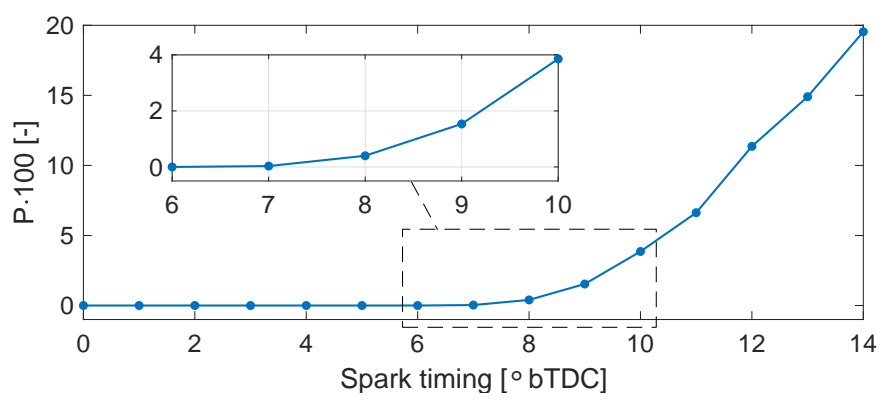

Figure 2. Average knock rate vs. fixed spark timing.

Knock events are binomially distributed regardless of the probability density of the knock intensity metric [26]. The latter assumption is widely acknowledged and it is true provided 
that the data are cycle-to-cycle uncorrelated. To prove that this assumption holds for the MAPO index, the autocorrelation of the knock occurrence, detected by comparing the MAPO index with a threshold, is shown in Figure 3. The analysis is performed on 200 cycles of data collected with a fixed spark timing of $14{ }^{\circ}$ bTDC. All the data are included in the $95 \%$ probability interval and thus it is reasonable to assume that the knock occurrence behaves as a cycle-to-cycle independent random process. Following the approach in [26], an engine

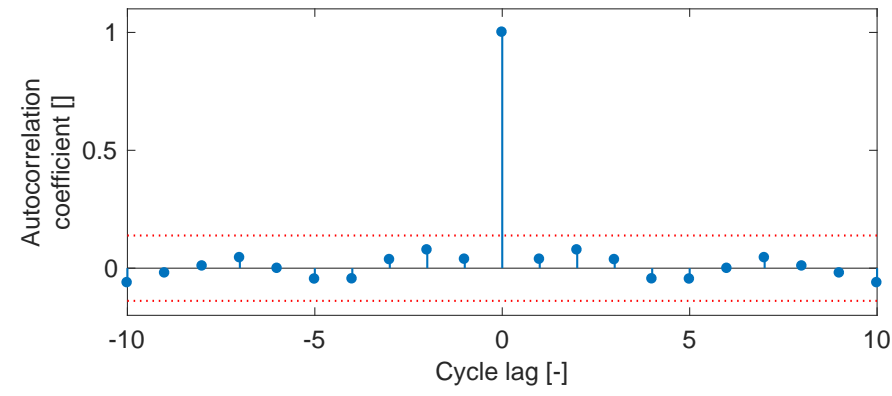

Figure 3. Autocorrelation of knock occurrence.

simulator based on a binomial distribution of knock events is built. This simulator is used in Section $\mathrm{V}$ for testing and comparing the knock control strategies.

\section{UNCONVENTIONAL KNOCK CONTROL}

The first proposed knock controller is based on the same principle as the conventional one: it advances the spark timing during non-knocking cycles and retards it at each knock event. The main novelty is the logarithmic advance law which allows to increase the average spark timing and to lower its variance. For this reason, the proposed strategy is called Unconventional.

\section{A. Conventional Strategy}

The conventional knock control strategy is implemented as in Equation (1)

$$
S_{c}(j)= \begin{cases}S_{c}(j-1)-K_{r e t} & \text { if knock, } \\ S_{c}(j-1)+K_{a d v} & \text { otherwise, }\end{cases}
$$

where $S_{c}(j)$ is the spark timing at cycle count $j, K_{\text {ret }}$ is the retarding quantity on knock events, and $K_{a d v}$ is the advancing quantity during non-knocking cycles. Under the assumption of stable operation (i.e., knock occurs deterministically at a fixed spark timing), the controller parameters (i.e., $K_{\text {ret }}$ and $K_{a d v}$ ) can be related to the target knock probability $\left(P_{\text {ref }}\right)$ using the following equation (see [26]):

$$
K_{a d v}=\frac{P_{r e f}}{1-P_{r e f}} K_{r e t} .
$$

While $P_{\text {ref }}$ is a design parameter related to the structural strength of the engine, the variable $K_{\text {ret }}$ can be considered as a control parameter and determines the reactivity of the controller. Large values of $K_{\text {ret }}$ allow for faster closed-loop transients but increase the variance and retard the average of the spark timing at steady-state operation. Therefore, $K_{\text {ret }}$ is normally manually tuned to find a suitable compromise between these two objectives.

\section{B. Unconventional Strategy}

While the conventional strategy advances the spark timing during non-knocking cycles at the constant rate $K_{a d v}$, the unconventional one uses a varying rate. The underlying idea is that, once the spark timing is retarded by $K_{r e t}$, the occurrence of consecutive knock events is rather improbable and thus the spark timing can be advanced faster. As the number of cycles from the last knock occurrence approaches $N_{\text {ref }}=\frac{1}{P_{\text {ref }}}$ the increase rate is reduced. Such behaviour can be implemented with a logarithmic function, as in the following equation:

$$
S_{u}(j)=\left\{\begin{array}{cc}
S_{u}(j-1)-K_{\text {ret }} & \text { if knock, } \\
S_{u}\left(j_{k}\right)+K_{l n} \ln (c) & \text { otherwise, }
\end{array}\right.
$$

where $K_{l n}$ is a tuning parameter, $j_{k}$ is the cycle count of the last knock event, $S_{u}\left(j_{k}\right)$ is the spark advance applied at the last knocking cycle, and $c$ is the number of cycles elapsed from the last knock event. The variable $K_{\text {ret }}$ here has the same function as for the conventional controller and, under the assumption of a stable operation, is related to $K_{l n}$ and the desired knock rate. Given $P_{r e f}$ and $K_{r e t}$, the value of $K_{l n}$ is determined by the following equation:

$$
K_{l n}=\frac{K_{r e t}}{\ln \left(\frac{1-P_{r e f}}{P_{r e f}}\right)} .
$$

Equation (4) ensures that the knocking spark timing is reached $N_{\text {ref }}$ cycles after the last knock event, corresponding to the desired probability. Figure 4 shows a typical spark timing evolution of the two controllers, assuming that knock events occur at $S_{k}=5{ }^{\circ}$ bTDC. Both controllers reach the knocking

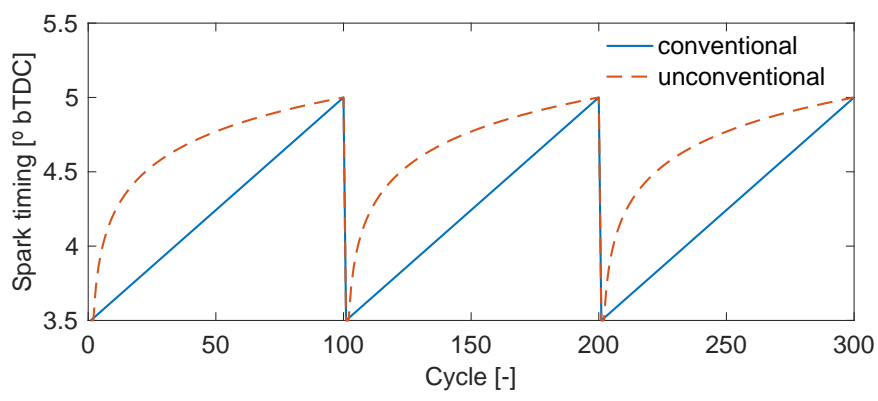

Figure 4. Unconventional vs. conventional controller evolution. $K_{\text {ret }}=1.5^{\circ}$, $P_{\text {ref }}=0.01$ and $N_{\text {ref }}=100$ cycles.

timing in $N_{\text {ref }}$ cycles, but the difference is in the shape used to advance the spark timing, which eventually determines the average and the variance of the control action.

\section{Deterministic Analysis}

Under the assumption of stable operation (i.e., evolutions as shown in Figure 4), the advantages of the unconventional controller can be computed analytically. The spark timing average and variance for the conventional controller are given by the following equations:

$$
E\left[S_{c}\right]=S_{k}-K_{r e t}+\frac{1}{N_{\text {ref }}} \sum_{j=0}^{N_{r e f}-1} S_{c}(j)=S_{k}-\frac{K_{r e t}}{2},
$$




$$
\operatorname{VAR}\left[S_{c}\right]=\frac{1}{N_{\text {ref }}} \sum_{0}^{N_{r e f}-1}\left(S_{c}(j)-E\left[S_{c}\right]\right)^{2}=\frac{K_{r e t}^{2}}{12},
$$

which have been derived by combining Equation (1) and (2). Analogously, one can derive the average and variance for the unconventional controller by combining Equation (3) and (4):

$$
\begin{aligned}
& E\left[S_{u}\right]=S_{k}-K_{r e t}+\frac{1}{N_{r e f}} \sum_{0}^{N_{r e f}-1} S_{u}(j)=S_{k}-\frac{K_{r e t}}{\ln \left(N_{r e f}\right)}, \\
& V A R\left[S_{u}\right]=\frac{1}{N_{\text {ref }}} \sum_{0}^{N_{r e f}-1}\left(S_{u}(j)-E\left[S_{u}\right]\right)^{2}=\frac{K_{r e t}^{2}}{\ln \left(N_{r e f}\right)^{2}} .
\end{aligned}
$$

As expected, higher values of $K_{\text {ret }}$ retards the average spark timing (i.e., reduces the engine efficiency) and increases its variance, for both approaches. The advantages of the unconventional controller are not evident since equations (7) and (8) depend on $N_{r e f}$. In particular, when $\ln \left(N_{r e f}\right)>2$, the unconventional controller outperforms the conventional one in terms of spark timing average and when $\ln \left(N_{\text {ref }}\right)^{2}>12$ in terms of its variance. The latter situation occurs for $N_{\text {ref }}$ bigger than 32, which means $P_{r e f}<0.03$. Considering that the typical range for $P_{r e f}$ found in the scientific literature is 0.001 to 0.02 , the use of the unconventional controller rather than the conventional is justified.

Besides steady-state properties, the settling time to recover from a steady-state operation deviation allows a dynamic comparison between the two approaches. The settling time required by the conventional and the unconventional algorithm to reach the knocking spark $S_{k}$ from a retarded condition $S(0)<S_{k}$ is given by Equations (9):

$$
T_{c}^{r e t}=\frac{1-P_{r e f}}{P_{r e f}} \cdot \frac{S_{d}}{K_{r e t}}, \quad T_{u}^{r e t}=\left(\frac{1-P_{r e f}}{P_{r e f}}\right)^{\frac{S_{d}}{K_{r e t}}}
$$

where $S_{d}=S_{k}-S(0)>0$. The settling time is non-trivially dependent on the reference probability, the controller parameter and the amplitude of the deviation. However, it can be clearly seen that it grows exponentially for the unconventional controller case as the ratio $\frac{S_{d}}{K_{\text {ret }}}$ increases; for the conventional controller such growth is only linear.

When dealing with advanced spark initializations, the time needed to reach steady state is equal (since both control strategies, (1) and (3), use the same retarding strategy) and is given by the following:

$$
T_{c}^{a d v}=T_{u}^{a d v}=\left\lceil\frac{-S_{d}}{K_{r e t}}\right\rceil,
$$

where $S_{d}<0$. Equation (10) allows to evidence that, for the usual values of $P_{\text {ref }}$ (such that $\frac{1-P_{\text {ref }}}{P_{\text {ref }}}>1$ ), the recovery time from an advanced condition is shorter than from a retarded one: therefore, both strategies are more reactive in response to dangerous than to low-efficiency conditions.

\section{AdAPTIVE KNOCK CONTROL}

The two controllers in Section III achieve the desired target probability $P_{r e f}$ and feature a design degree of freedom, namely the value of the parameter $K_{\text {ret }}$. Large values of $K_{\text {ret }}$ would be preferable for conditions distant from the target one, since they yield faster recovery transients (see e.g. (9)), whereas small values of $K_{r e t}$ would result in a lower steadystate variance when the probability target is met.

The idea of the adaptive strategy proposed here is to estimate the difference between the actual and the desired stochastic properties of knock occurrences and to change the $K_{\text {ret }}$ value accordingly. Differently from other studies, e.g. [20], that develop "fully" stochastic controllers, here the stochastic information of knock events is used to adapt the parameters of deterministic controllers rather than to directly compute the control action.

The adaptive controller scheme is shown in Figure 5 and applies equally to the conventional and the unconventional controller. In the proposed approach, the likelihood ratio

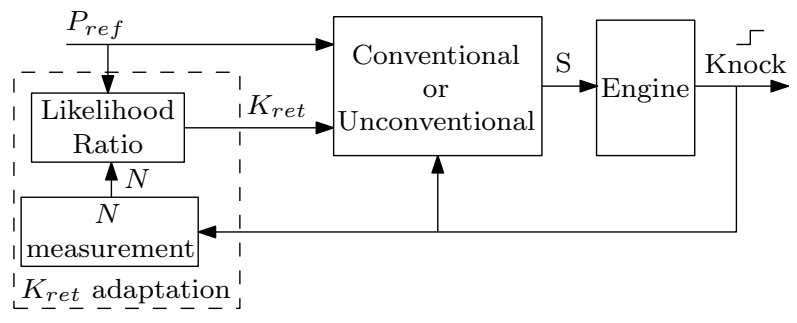

Figure 5. Adaptive knock control scheme.

$\mathcal{L}_{r}$ is used as an indicator of the discrepancy between the expected and the measured stochastic properties of knock. It is computed according to the following expression:

$$
\mathcal{L}_{r}=\frac{\left(P_{\text {ref }}\right)^{N_{k}}\left(1-P_{\text {ref }}\right)^{N-N_{k}}}{\left(P_{\text {meas }}\right)^{N_{k}}\left(1-P_{\text {meas }}\right)^{N-N_{k}}},
$$

where $N_{k}$ is a designed parameter which defines the number of past knock events considered, $N$ is the number of past cycles in which $N_{k}$ events occurred, $P_{\text {meas }}=N_{k} / N$ is the sampled and $P_{r e f}$ is the target probability. The likelihood ratio compares the measured probability of obtaining $N_{k}$ events in $N$ samples with those of a binomial stochastic process with parameter $P_{r e f}$. For instance, given a desired probability of 0.01 , the likelihood ratio is greatest when the actual distance between the last two knock events $\left(N_{k}=1\right)$ is equal to 100 cycles, or the last three events $\left(N_{k}=2\right)$ occur in 200 cycles. As such, $N_{k}$ plays the role of a filtering parameter and defines the memory of the update mechanism: higher values imply a larger time window considered for the computation of $N$ and $P_{\text {meas }}$. In the simulations and experiments that follow, a fixed value of $N_{k}=1$ is used; larger values would reduce the variability of the parameter adaptation at the cost of a longer settling time.

The adaptation strategy increases $K_{\text {ret }}$ when the stochastic properties of knock occurrences are different from those desired (i.e. when $\mathcal{L}_{r}<1$ ), increasing the convergence speed of the deterministic controllers to the target values. As the desired knock rate is met (i.e. when $\mathcal{L}_{r} \rightarrow 1$ ), $K_{\text {ret }}$ is driven smaller values so as to obtain better steady-state properties. To avoid extreme values of the $K_{r e t}$ it is saturated between 
a maximum $\left(K_{r e t}^{\max }\right)$ and a minimum $\left(K_{\text {ret }}^{\min }\right)$ value. Equation (12) implements the parameter adaptation:

$$
K_{\text {ret }}=K_{\text {ret }}^{\min }+\left(K_{\text {ret }}^{\max }-K_{\text {ret }}^{\min }\right)\left(1-\mathcal{L}_{r}\right),
$$

By definition, the likelihood ratio (11) is updated only when a knock event is measured. Thus, the adaptation of $K_{\text {ret }}$ occurs at a lower speed than the cycle-by-cycle spark timing update. This different time scale explains the use of the term adaptive to address the update parameter mechanism (12). It should be remarked that it is not necessary to update the value of $\mathcal{L}_{r}$ during non-knocking cycles, since the spark advance increase during non-knocking cycles, which is a feature of the deterministic controllers, guarantees that a knock event is eventually triggered. The same could not be done for the benchmark controller, which relies on $\mathcal{L}_{r}$ for the computation of the spark timing.

The adaptive strategies have three tuning parameters. $P_{r e f}$ and $K_{r e t}^{\max }$ are equivalent to $P_{r e f}$ and $K_{r e t}$ of the non-adaptive strategies. The value of $K_{r e t}^{\min }$ modifies how the adaptation strategy works: when $K_{r e t}^{\min }=K_{r e t}^{\max }$, no adaptation is performed, whereas $K_{r e t}^{\min }=0$ is the minimum tuning value that keeps the spark constant when the reference probability is met. An intermediate value of $K_{r e t}^{m i n}$ should be chosen based on the minimum controller reaction speed desired.

\section{Simulation Results}

In this section, the proposed controllers are compared with the conventional strategy and the stochastic benchmark controller discussed in [20], which updates the control variable proportionally to the error between the actual likelihood ratio and a target threshold $\mathcal{L}_{r, t h}$. Unlike the conventional strategy, the variables $K_{a d v}$ and $K_{r e t}$ are not related to the reference probability, which makes the tuning process more difficult. The Algorithm 3 version of the benchmark controller is used here, which improves the transient response after long periods of operation at the desired target.

The comparison is performed using the stochastic knock simulator [26]. Steady-state performances are considered in Section V-A and a specific analysis of the adaptation strategy tuning is discussed in Section V-B.

\section{A. Steady-State Operation}

The reference probability for the steady-state comparison is set at 0.01 , consistent with application realistic values; the parameters of all the tested controllers are summarized in Table I. It should be noted that in order to equate the maximum reaction speed for the adaptive and non-adaptive strategies, $K_{r e t}^{\max }$ and $K_{\text {ret }}$ have the same value. The values of $K_{\text {ret }}$ and $K_{a d v}$ of the benchmark controller are tuned to obtain an average knock rate of approximately 0.01 , while the threshold $\mathcal{L}_{r, t h}$ is set according to the reference paper suggestions.

The first cycles of a simulation are shown in Figure 6, where each of the discussed controller is compared with the conventional one. The non-adaptive controllers have the most straightforward behaviour: they both retard the timing on knock events and advance it otherwise. The conventional
Table I

CONTROLLER PARAMETERS FOR THE STEADY-STATE COMPARISON

\begin{tabular}{cccccc}
\hline & conv & unconv & ad conv & ad unconv & bench \\
\hline$P_{\text {ref }}$ & 0.01 & 0.015 & 0.01 & 0.015 & 0.01 \\
$K_{\text {ret }}$ & $1.5^{\circ}$ & $1.5^{\circ}$ & online & online & $0.5^{\circ}$ \\
$K_{\text {ret }}^{\text {max }}$ & - & - & $1.5^{\circ}$ & $1.5^{\circ}$ & - \\
$K_{\text {ret }}^{\text {min }}$ & - & - & $0.1^{\circ}$ & $0.1^{\circ}$ & - \\
$K_{a d v}$ & 0.015 & - & online & - & $1.25^{\circ}$ \\
$K_{l n}$ & - & 0.3264 & - & online & - \\
$\mathcal{L}_{r, t h}$ & - & - & - & - & 0.4 \\
\hline
\end{tabular}

controller advances the timing linearly, while the unconventional controller advances it logarithmically. The benchmark controller - being a fully stochastic controller - does not react at each cycle and it changes the spark timing only when the likelihood ratio overcomes the threshold. The adaptive strategies retard the timing depending on the discrepancy between the expected and the measured cycles between two consecutive knock events: this is the reason for the different amplitude of the retarding action at each knock event.
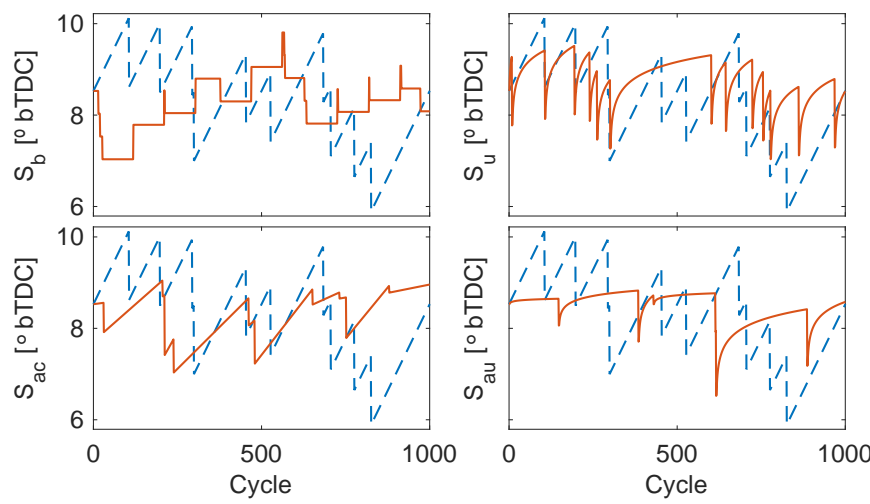

Figure 6. Simulation example at steady-state operation. In each subplot, the conventional controller (dashed line) is compared with the benchmark (top left), the unconventional (top right), the adaptive conventional (bottom left) and the adaptive unconventional controller (bottom right).

The controllers were simulated for 25000 cycles and the average results are shown in Table II. The proposed controllers outperform both the conventional and the benchmark ones. While the improvement in terms of average spark timing (i.e., engine efficiency) is limited by the engine characteristics, the improvement in terms of spark timing variability is considerable. In this regard, the adaptive unconventional controller shows the best performance.

Table II

SIMULATION RESULTS AT STEADY-STATE OPERATION

\begin{tabular}{cccccc}
\hline & conv & unconv & ad conv & ad unconv & bench \\
\hline$E[P] \cdot 100(-)$ & 1 & 0.99 & 1.04 & 1.01 & 1.04 \\
$E[S]\left({ }^{\circ}\right)$ & 8.35 & 8.48 & 8.64 & 8.56 & 8.30 \\
$V A R[S]\left({ }^{\circ} 2\right)$ & 0.71 & 0.30 & 0.30 & 0.13 & 0.37 \\
\hline
\end{tabular}

An important aspect that must be highlighted is the discrepancy between the desired knock rate and the resulting one (see Figure 7). Within this perspective, the conventional controllers show the best matching whereas the unconventional and the benchmark ones require an appropriate parameter tuning to 
match the desired rate. However, while the unconventional strategies require only the tuning of $P_{r e f}$, the parameters of the benchmark controller are coupled, which complicates their tuning.

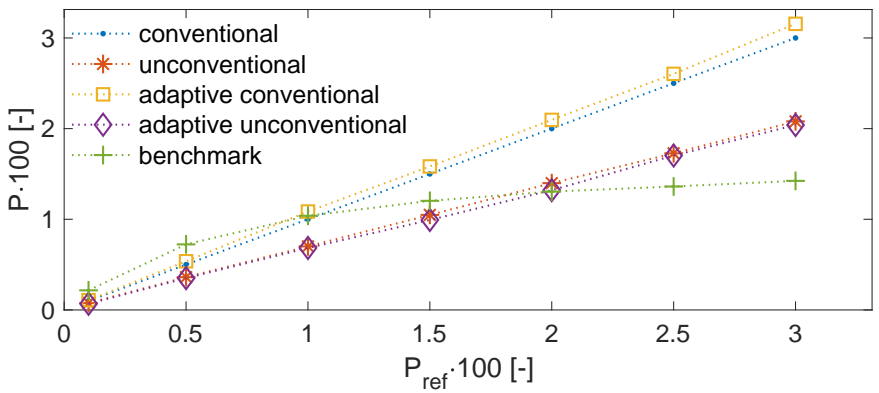

Figure 7. Actual knock rate $(P)$ vs. reference probability $\left(P_{r e f}\right)$. The other parameters are equal to those listed in Table I.

\section{B. Stochastic Adaptation Tuning}

This section is devoted to the analysis of the tuning of the adaptive strategies. In fact, $K_{\text {ret }}^{\max }$ and $K_{\text {ret }}^{\text {min }}$ determine the maximum and the minimum reactivity of the adaptive controllers. The objective is thus to explore the possible trade-offs between the controller speed and the spark timing variability. In order to do so, the adaptive controllers are compared to the respective non-adaptive versions for a fixed value of $P_{\text {ref }}$ and different values of $K_{\text {ret }}, K_{r e t}^{\max }$ and $K_{r e t}^{\mathrm{min}}$. However, $K_{\text {ret }}$ and $K_{\text {ret }}^{\max }$ are always intentionally chosen to be equal in order to have the maximum speed of the adaptive strategies equal to the speed of the non-adaptive ones.

Figure 8 shows the sensitivity results w.r.t. $K_{\text {ret }}$ and $K_{\text {ret }}^{\max }$, obtained by averaging 1000 simulations. The notable effect is that by changing $K_{r e t}$ and $K_{r e t}^{\max }$, it is possible to achieve settling times that are as short as those of the conventional controller while reducing the variability of the spark timing by more than $50 \%$.

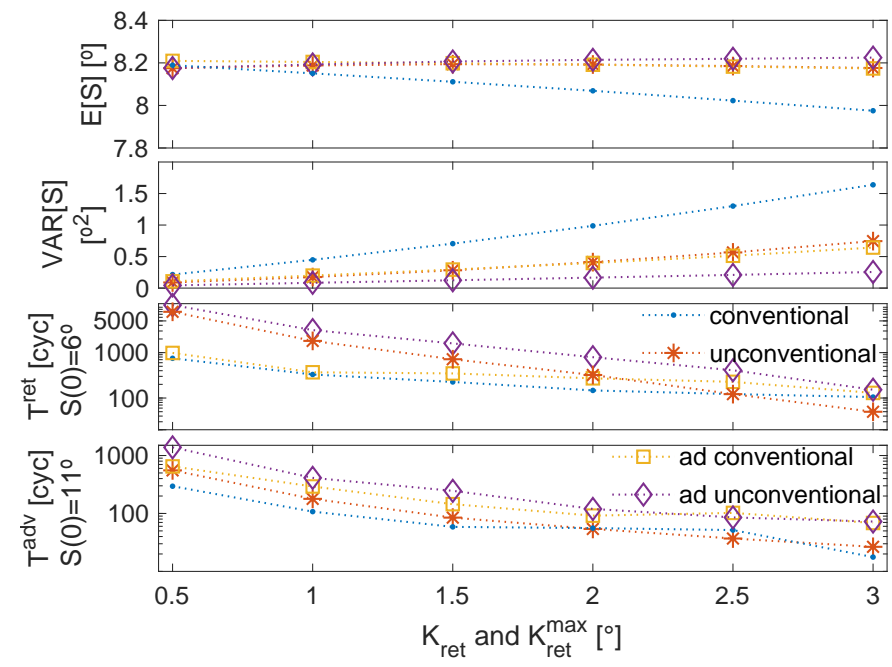

Figure 8. Sensitivity to $K_{\text {ret }}$ and $K_{\text {ret }}^{\max }$ (the other parameters are equal to those listed in Table I).

The action of the adaptive strategy depends also on the value of $K_{r e t}^{\min }$, which determines the amplitude of the adaptation (i.e., the variation range of $K_{r e t}$ ). The averaged results of the sensitivity w.r.t. this parameter are shown in Figure 9. As expected, when the value of $K_{\text {ret }}^{\text {min }}$ approaches $K_{\text {ret }}^{\text {max }}$, the effect of the adaptive rule vanishes and the adaptive controllers behave similarly to their non-adaptive counterparts. Low values of $K_{r e t}^{m i n}$ yield better stochastic performances at the cost of an increase of the settling time in response to a wrong initialization.

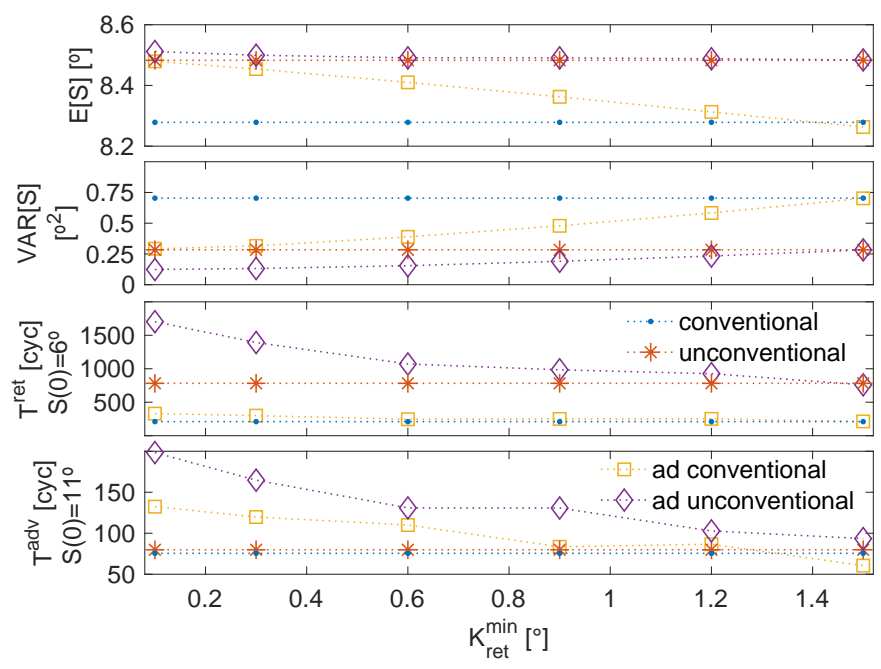

Figure 9. Sensitivity to $K_{r e t}^{\min }$ (the other parameters are equal to those listed in Table I).

\section{EXPERIMENTAL RESULTS}

The performances of the controllers are experimentally compared at a test bench composed of an electric brake and a four-stroke three-cylinders SI engine. The engine is a 499.6 cc ( $82 \mathrm{~mm}$ bore and 10.1:1 compression ratio) turbocharged with variable valve camshaft and direct gasoline injection. The injection timing is set at $270{ }^{\circ}$ bTDC and the intake valve closing was set at $180^{\circ} \mathrm{bTDC}$. The air-to-fuel ratio is measured by a sensor at the exhaust and is regulated at stoichiometric conditions by closed-loop-controlling the amount of fuel injected: this allows a validation of the proposed control strategies in a realistic situation, when also other closed-loop controllers are active. The air mass flow is measured by a hotfilm anemometer and controlled by a waste-gate valve located before the turbocharger inlet.

All the tests are performed at a speed of $1500 \mathrm{rpm}$, an air mass flow equal to $667 \mathrm{mg} / \mathrm{stroke}$, a coolant temperature of $85^{\circ} \mathrm{C}$, and a rail pressure of 200 bar.

Steady-state and transitory performances have been analysed; each controller features the same tuning parameters as those used for the simulations.

\section{A. Steady-State Operation}

The average results of the steady-state experiments $(25000$ cycles) are summarized in Table III and confirm those obtained in the simulations: the adaptive strategies present the best behaviour with the most advanced timing and the lowest variance. Besides the relative strategy comparison, it is worth 
noting how the absolute performances of the controllers are close the simulation ones.

Table III

EXPERIMENTAL RESULTS AT STEADY-STATE OPERATION

\begin{tabular}{cccccc}
\hline & conv & unconv & ad conv & ad unconv & bench \\
\hline$E[P] \cdot 100(-)$ & 1 & 1.08 & 1.01 & 1.07 & 1.04 \\
$E[S]\left({ }^{\circ}\right)$ & 8.22 & 8.27 & 8.51 & 8.47 & 8.13 \\
$V A R[S]\left({ }^{\circ}\right)$ & 0.71 & 0.29 & 0.40 & 0.11 & 0.44
\end{tabular}

\section{B. Transitory Behaviour}

In the experimental analysis of the transitory behaviour of the controllers, the settling time when starting from a wrong spark advance initialization is evaluated. In the present context, such time is referred to as the number of cycles required to reach steady-state conditions. Since the target knock rate is 0.01 , according to the stationary engine relation (Figure 2 ), the expected average spark timing at the 0.01 rate is $8.53^{\circ} \mathrm{bTDC}$ and steady-state conditions are considered to be reached when the spark advance reaches such value for the first time. Since all controllers advance and retard the spark timing differently, both scenarios are considered and the spark advance is initialized $2^{\circ}$ away from the expected steady-state value.

Figure 10 and 11 show examples of the controller responses starting from the two conditions. Considering the stochastic
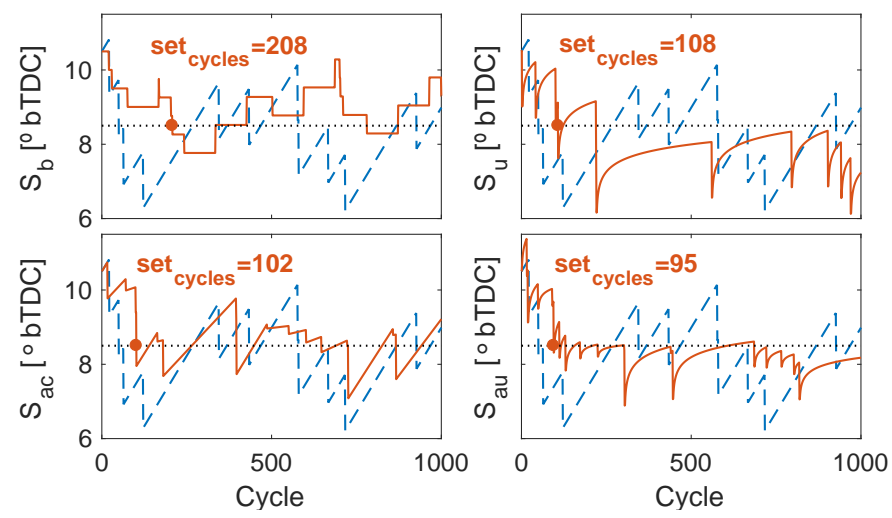

Figure 10. Evolution of the spark timing starting from advanced condition. In each subplot the conventional controller (dashed line) is compared with the benchmark (top left), the unconventional (top right), the adaptive conventional (bottom left), and the adaptive unconventional controllers (bottom right).

nature of knock, the results of the settling time may vary for one experiment to another. Thus, in order to better evaluate the differences among the controllers, 10 experiments have been performed for both starting conditions and the average results collected in Table IV.

Among the proposed controllers, the adaptive conventional one proves to be the fastest: while its settling times are longer than those of the conventional controller, it outperforms the stochastic benchmark controller.

The unconventional controller presents a twofold behaviour: its settling time when starting from advanced conditions is small, almost as the adaptive conventional controller's one but its long when starting from a retarded condition. When
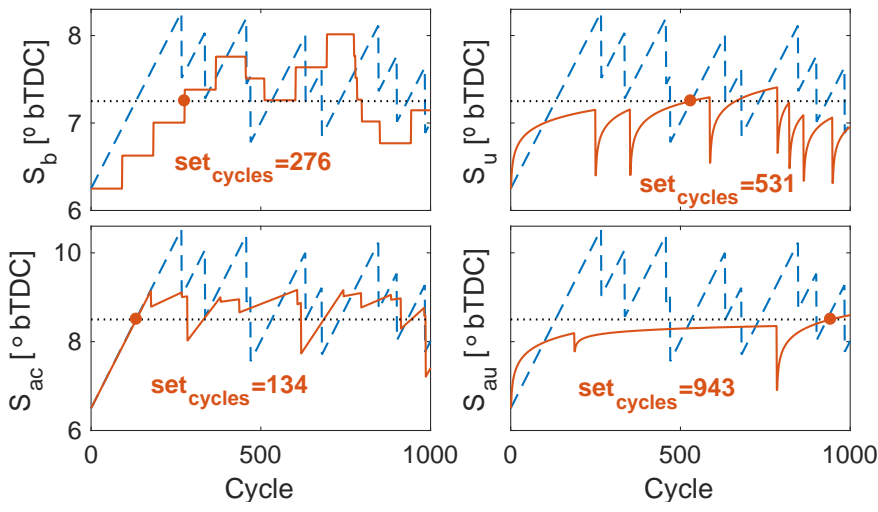

Figure 11. Evolution of the spark timing starting from retarded condition. In each subplot the conventional controller (dashed line) is compared with the benchmark (top left), the unconventional (top right), the adaptive conventional (bottom left), and the adaptive unconventional controllers (bottom right).

considering both conditions, the adaptive unconventional is the slowest one. Therefore, the unconventional controllers are most suitable for steady-state conditions, whereas the adaptive conventional controller shows an excellent trade-off between variability and convergence speed.

Finally, it is also worth noting how the experimental results match those of the deterministic analysis in Section III.

Table IV

RESULTS OF SETTLING TIME ANALYSIS (10 EXPERIMENTS)

\begin{tabular}{lcc}
\hline Strategy & Advanced start & Retarded start \\
\hline Conventional & 53 cycles & 162 cycles \\
Unconventional & 102 cycles & 523 cycles \\
Adaptive conventional & 106 cycles & 287 cycles \\
Adaptive unconventional & 181 cycles & 907 cycles \\
Benchmark & 249 cycles & 328 cycles \\
\hline
\end{tabular}

\section{CONCLUSION}

In this paper, the knock control problem has been addressed introducing three innovative controllers. The unconventional controller uses a logarithmic shape to increase the spark timing; the adaptive conventional and unconventional controllers adapt their parameters according to the likelihood ratio, hence combining the advantages of deterministic and stochastic approaches to the knock control. Besides a wellknown conventional strategy, the proposed controller have been compared with a stochastic state-of-the-art solution.

Experimental results at the test bench show how the logarithmic advance of the spark timing allows to get the lowest variance at steady-state. However, when the transient response is considered, the conventional controller still shows the best results. Therefore, the adaptive conventional strategy is the most promising as it features an excellent compromise between response time and steady-state behaviour.

Apart from their good performance, all the proposed strategies require fewer tuning parameters and lower effort compared to the benchmark controller. In addition, it must be remarked how the simulation analysis using the stochastic simulator allows a realistic design of the proposed strategies, which yielded to consistent results at the engine test bench with no additional empirical tuning effort. 


\section{REFERENCES}

[1] L. Guzzella and C. Onder, Introduction to Modeling and Control of Internal Combustion Engine Systems, 2nd ed. Springer-Verlag Berlin Heidelberg, 2010.

[2] E. Pipitone and A. Beccari, "A study on the use of combustion phase indicators for mbt spark timing on a bi-fuel engine," SAE Technical Paper, Tech. Rep., 2007.

[3] S.-H. Jang, Y.-G. Lee, T.-Y. Oh, and K.-S. Park, "An experimental study on knock sensing for a spark ignition engine," in SAE Technical Paper. SAE International, November 1993.

[4] K. Burgdorf and I. Denbratt, "Comparison of cylinder pressure based knock detection methods," in SAE Technical Paper. SAE International, October 1997.

[5] S. Ortmann, M. Rychetsky, M. Glesner, R. Groppo, P. Tubetti, and G. Morra, "Engine knock estimation using neural networks based on a real-world database," in SAE Technical Paper. SAE International, February 1998.

[6] E. Pipitone and L. D'Acquisto, "Development of a low-cost piezo film-based knock sensor," Proceedings of the Institution of Mechanical Engineers, Part D: Journal of Automobile Engineering, vol. 217, no. 8, pp. 691-699, 2003.

[7] J. Naber, J. R. Blough, D. Frankowski, M. Goble, and J. E. Szpytman, "Analysis of combustion knock metrics in spark-ignition engines," in SAE Technical Paper. SAE International, April 2006.

[8] J. M. Borg, G. Saikalis, S. Oho, and K. C. Cheok, "Knock signal analysis using the discrete wavelet transform," in SAE Technical Paper. SAE International, April 2006.

[9] M. Ettefagh, M. Sadeghi, V. Pirouzpanah, and H. A. Tash, "Knock detection in spark ignition engines by vibration analysis of cylinder block: A parametric modeling approach," Mechanical Systems and Signal Processing, vol. 22, no. 6, pp. 1495-1514, 2007.

[10] G. Wu, "A real time statistical method for engine knock detection," in SAE Technical Paper. SAE International, April 2007.

[11] Y. Lonari, C. Polonowski, J. Naber, and B. Chen, "Stochastic knock detection model for spark ignited engines," in SAE Technical Paper. SAE International, April 2011.

[12] E. Galloni, "Dynamic knock detection and quantification in a spark ignition engine by means of a pressure based method," Energy Conversion and Management, vol. 64, pp. 256-262, 2012.

[13] P. Bares, D. Selmanaj, C. Guardiola, and C. Onder, "Knock probability estimation through an in-cylinder temperature model with exogenous noise," Mechanical Systems and Signal Processing, vol. 98, pp. 756769, 2018.

[14] K. Akimoto, H. Komatsu, and A. Kurauchi, "Development of pattern recognition knock detection system using short-time fourier transform," IFAC Proceedings Volumes, vol. 46, no. 21, pp. 366-371, 2013.

[15] U. Kiencke and L. Nielsen, Automotive control systems: for engine, driveline, and vehicle. Springer Science \& Business Media, 2005.

[16] M. Penese, C. F. Damasceno, A. Bucci, and G. Montanari, "Sigma® on knock phenomenon control of flexfuel engines," in SAE Technical Paper. SAE International, November 2005, p. 3990.

[17] N. Cavina, G. Po, and L. Poggio, "Ion current based spark advance management for maximum torque production and knock control," in ASME 8th Biennial Conference on Engineering Systems Design and Analysis. American Society of Mechanical Engineers, 2006, pp. 537545.

[18] G. Zhu, I. Haskara, and J. Winkelman, "Stochastic limit control and its application to spark limit control using ionization feedback," in Proceedings of the 2005, American Control Conference, 2005. IEEE, June 2005, pp. 5027-5034.

[19] J. C. P. Jones, J. Frey, and K. R. Muske, "A fast-acting stochastic approach to knock control," IFAC Proceedings Volumes, vol. 42, no. 26, pp. 16-23, 2009.

[20] J. C. P. Jones, J. M. Spelina, and J. Frey, "Likelihood-based control of engine knock," IEEE Transactions on Control Systems Technology, vol. 21, no. 6, pp. 2169-2180, November 2013.

[21] A. Thomasson, H. Shi, T. Lindell, L. Eriksson, T. Shen, and J. C. P. Jones, "Experimental validation of a likelihood-based stochastic knock controller," IEEE Transactions on Control Systems Technology, vol. 24, no. 4, pp. 1407-1418, July 2016.

[22] U. Lezius, M. Schultalbers, W. Drewelow, and B. Lampe, "Improvements in knock control," in Mediterranean Conference on Control \& Automation, 2007. MED'07. IEEE, June 2007, pp. 1-5.

[23] G. Panzani, C. Onder, and F. Ostman, "Engine knock margin estimation using in-cylinder pressure measurements," IEEE/ASME Transactions on Mechatronics, pp. 1-12, 2016.
[24] A. J. Shahlari and J. B. Ghandhi, "A comparison of engine knock metrics," in SAE Technical Paper. SAE International, October 2012.

[25] P. Bares, D. Selmanaj, C. Guardiola, and O. Christopher, "A new knock event definition for knock detection and control optimization," Applied Thermal Engineering, vol. 131, pp. 80-88, 2018.

[26] J. M. Spelina, J. C. P. Jones, and J. Frey, "Stochastic simulation and analysis of a classical knock controller," International Journal of Engine Research, vol. 16, no. 3, pp. 461-473, 2015. 